\title{
Electric-field-induced phase transitions in bent-core mesogens determined by $x$-ray diffraction
}

\author{
J. Ortega,,${ }^{1, *}$ C. L. Folcia, ${ }^{2}$ J. Etxebarria, ${ }^{2}$ J. Martínez-Perdiguero, ${ }^{2, \dagger}$ J. A. Gallastegui, ${ }^{3,4}$ P. Ferrer, ${ }^{3,4}$ N. Gimeno, ${ }^{5}$ and \\ M. Blanca Ros ${ }^{5}$ \\ ${ }^{1}$ Física Aplicada II, FCT/ZFT, UPV/EHU, Apartado 644, E-48080 Bilbao, Spain \\ ${ }^{2}$ Física de la Materia Condensada, FCT/ZFT, UPV/EHU, Apartado 644, E-48080 Bilbao, Spain \\ ${ }^{3}$ SpLine Spanish CRG beamline at the European Synchrotron Radiation Facility, BP 220, FR-38043 Grenoble Cedex 9, France \\ ${ }^{4}$ Instituto de Ciencia de Materiales de Madrid, Consejo Superior de Investigaciones Científicas, Sor Juana Inés de la Cruz, 3 , \\ ES-28049 Madrid, Spain \\ ${ }^{5}$ Departamento de Química Orgánica, Instituto de Ciencia de Materiales de Aragón (ICMA), Facultad de Ciencias, \\ Universidad de Zaragoza-CSIC, E-50009 Zaragoza, Spain
}

(Received 15 April 2011; published 23 August 2011)

\begin{abstract}
The changes in the x-ray diffraction diagram induced by an electric field have been studied in four bent-core liquid crystals. Two of the compounds present columnar $\mathrm{B}_{1}$ phases and the other two show dark conglomerate (DC) structures. In the $\mathrm{B}_{1}$ materials the electric field gives rise to switchable phases whose diffraction pattern is that of the smectic $\mathrm{C}$ polar $(\mathrm{SmCP})$ phase. In the $\mathrm{DC}$ structures the positions of the Bragg reflections remain essentially unaltered, but in one of the compounds the peaks become narrower under field, indicating that the correlation length of the smectic order increases. A slight enhancement of the smectic spacing is found in the DC phases upon field application.
\end{abstract}

DOI: 10.1103/PhysRevE.84.021707

PACS number(s): 61.30.-v, 61.05.cf, 64.70.mj

\section{INTRODUCTION}

Bent-core compounds have been one of the most studied topics among liquid crystals in the last years. These materials are composed of bent-shaped molecules whose self-interaction gives rise to a rich variety of mesophases [1-3]. The most outstanding features they present are ferroelectricity, with high polarization values, and chirality, even with achiral molecules. The molecules can self-organize in a wide variety of arrangements, many of them exclusive of this type of molecule. Among them there are two-dimensional (2D) periodic phases, exotic structures such as the dark conglomerate (DC) phase, or the still mysterious $\mathrm{B}_{4}$ phase. Figure 1 shows a schematic representation of a bent-core molecule and some phases often found in these compounds.

During recent years great progress in the understanding of the physics of bent-core liquid crystals has been achieved, though many issues about their structural properties still require further research. In this work we will focus on some phase transitions induced by electric fields that are observed in this kind of material. As a general rule, the electric field promotes a more effective molecular packing of the bent-core molecules, which, supposedly, segregate in smectic layers. Therefore, columnar $\mathrm{B}_{1}$ mesophases, or other modulated or distorted smectic phases can be destabilized by the electric field to form flat smectic layers. Here we will deal specifically with the $\mathrm{B}_{1}-\mathrm{SmCP}$ and DC-SmCP transitions induced by an electric field.

Contrary to the SmCP (also called $\mathrm{B}_{2}$ ) phases, the columnar $\mathrm{B}_{1}$ phase does not respond to the electric field in most cases. The molecular confinement inside the blocks that

\footnotetext{
*Corresponding author: josu.ortega@ehu.es

${ }^{\dagger}$ Present address: Departamento de Micro y Nanotecnologia, Tekniker, E-20600 Eibar, Spain
}

constitute the columns (see Fig. 1) hinders the reorientation of the molecules under field. However, in some cases, high enough fields can overcome this barrier and give rise to a phase transition that is believed to favor the formation of smectic layers against blocks. In this way, the structural restrictions to the molecular switching are eliminated. The response to the field may depend on the nature of the $B_{1}$ phase and, in particular, the molecular tilt seems to be a relevant parameter. For example, in the nontilted $\mathrm{B}_{1}$ phase only a response due to the dielectric anisotropy is observed [4]. On the other hand, the switching performance often depends on the frequency and shape of the applied voltage. There are several examples of this behavior reported in the literature [5-7]. In the majority of them the phase transition is detected on the basis of texture observation. However, the identification of the field-induced phases is usually hard to accomplish since the domain size of the textures is often very small. X-ray scattering measurements are evidently a more reliable alternative to determine the structure of the phases. Unfortunately, very few examples of this kind of study have appeared in the literature, probably due to the inherent experimental difficulties of performing the $\mathrm{x}$-ray experiment while applying electric field to the samples. In $[4,8]$ only an electric-field-induced reorientation of the molecules is observed, but the material maintains the $\mathrm{B}_{1}$ structure upon field application. In [9] the peaks that can be indexed in terms of a $2 \mathrm{D}$ structure do not completely disappear under field.

The second point studied in the present work is the behavior of the DC phase under field. This phase appears quite often in bent-core compounds and exhibits very interesting and uncommon features [10-14], which could even be used for display applications [15]. It shows short-range orientational order, but macroscopically is isotropic. In fact, the structure presents smectic arrangement but the layers are bent at a mesoscopic scale forming a spongelike structure (see Fig. 1). 


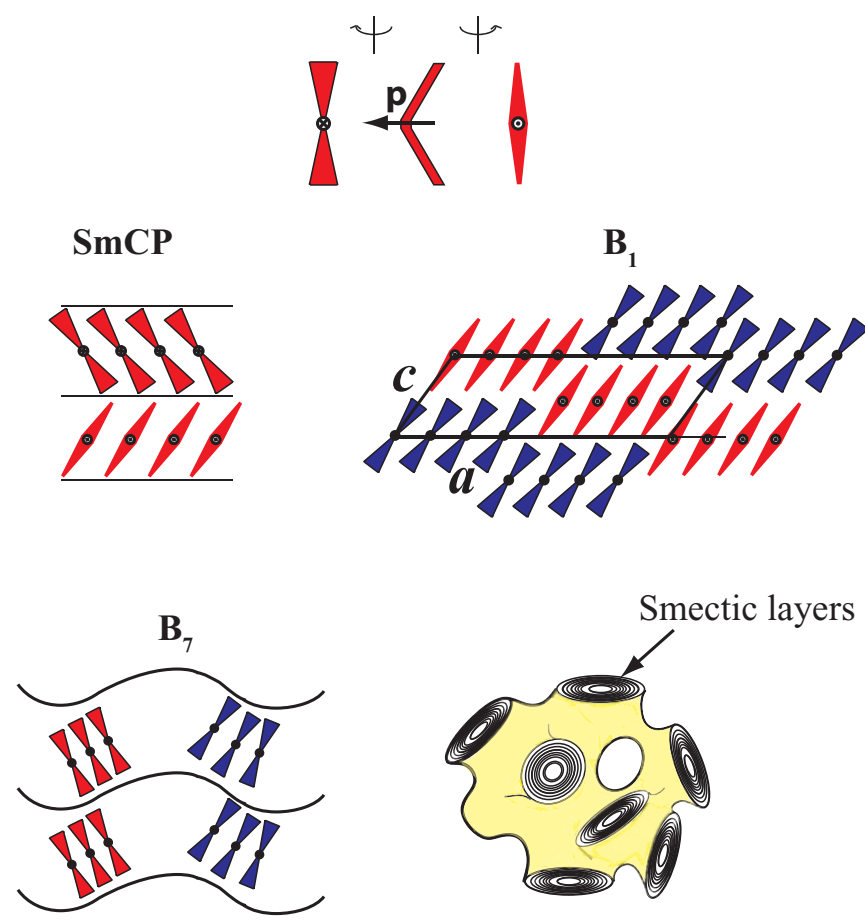

Dark Conglomerate

FIG. 1. (Color online) Schematic representation of a bent-shaped molecule depending on the viewing angle together with some of the most frequently observed bent-core mesophases.

This layer deformation is due to an intralayer structural mismatch because of the different tilt orientation of the wings of the molecule, which results in a saddle-splay curvature. Under electric field this phase becomes birefringent and usually presents a non-well-defined texture that has been claimed to be the SmCP phase. For this case, to the best of our knowledge, not a single example of an x-ray study exists in the literature.

In this paper we present an X-ray analysis of four bentcore materials showing transitions under field. Two of them have a $\mathrm{B}_{1}$ ground state while the other two are DC phases. The diffraction patterns with and without field are compared. The experiments require the use of an intense $\mathrm{x}$-ray source since the samples are sandwiched between glass plates and the amount of material is relatively small.

\section{EXPERIMENTAL}

X-ray diffraction measurements in transmission mode were carried out at a fixed wavelength of $0.95 \AA$ on a six-circle diffractometer at SpLine beamline (BM25B), ESRF, Grenoble, France [16]. A point-scintillation and a 2D charge coupled device (CCD) detectors were used. Measurements were performed using homemade samples of $10-\mu \mathrm{m}$ thickness sandwiched between ITO coated glass plates as electrodes. The substrates had a thickness of $100 \mu \mathrm{m}$, and no chemical treatment was used, since bent-core molecules do not align by the rubbing process. Therefore, for x-ray purposes the samples can be considered as powder. The cells were placed on a hot stage with $0.1{ }^{\circ} \mathrm{C}$ temperature control.

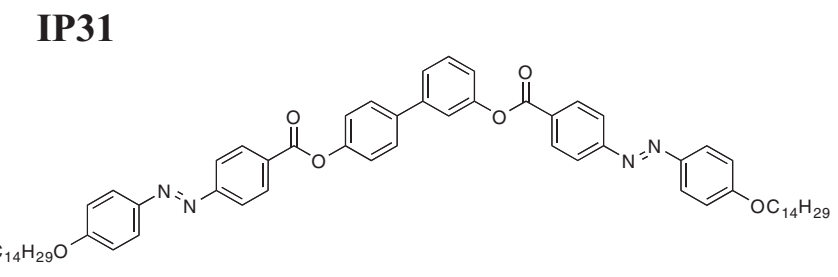

\section{Iso- $161^{\circ} \mathrm{C}-\mathbf{B}_{1}-128^{\circ} \mathrm{C}-$ Crystal}

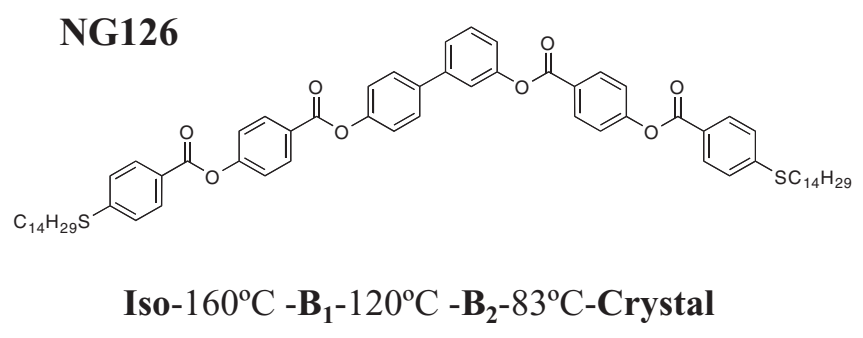

FIG. 2. Chemical structure and phase sequence of the compounds showing the columnar $\mathrm{B}_{1}$ structure.

\section{FIELD-INDUCED $B_{1}-B_{2}$ PHASE TRANSITION}

Figure 2 presents the chemical structure and phase sequence of IP31 and NG126 compounds. A characterization of these materials is reported in Refs. [17,18] and in Refs. [5,6,19], respectively. In both compounds a field-induced $\mathrm{B}_{1}-\mathrm{B}_{2}$ phase transition was proposed on the basis of texture examination. This transition was also predicted theoretically by the polarization-splay model of Ref. [20].

Figures 3(a) and 3(c) show the textures of the $B_{1}$ phase of IP31 and NG126, respectively, observed in cells of $5 \mu \mathrm{m}$ thickness. Typical domain characteristic of this phase can be observed (banana-leaf domains). On applying an electric field, the domains break and the birefringence increases. The transition seems to be abrupt with a threshold field of the order of $10 \mathrm{~V} / \mu \mathrm{m}$ as reported in [5]. Figures 3(b) and 3(d) show the textures under an electric field of $15 \mathrm{~V} / \mu \mathrm{m}$ perpendicular to the substrate.

Solid circles in Fig. 4(a) represent the X-ray diagram of IP31 at $145^{\circ} \mathrm{C}$ without electric field. The data were obtained by azimuthal integration of the signal at the CCD camera. The obtained peaks can be indexed on the basis of a centered oblique lattice as (11), (11), (02), and (22) [21]. In Fig. 1 the used unit cell is schematically depicted. It must be pointed out that our experiment does not distinguish between opposite polarization directions.

Under an electric field of $14 \mathrm{~V} / \mu \mathrm{m}$ the diffraction pattern is clearly modified [open circles in Fig. 4(a)]. As can be seen, only two peaks remain. They can be indexed, assuming a smectic structure, as (01) and (02). In addition, it is evident that the (11) reflection in the $\mathrm{B}_{1}$ phase, which corresponds to the pseudolayer periodicity (see Ref. [22]), gives rise to the (01) peak under field application. Therefore, the electric field does promote the formation of the smectic layers from the pseudolayers composed by blocks.

In the case of this compound, texture observation only allows the identification of the existence of the SmCP phase if the sample is cooled down from the isotropic liquid to the 

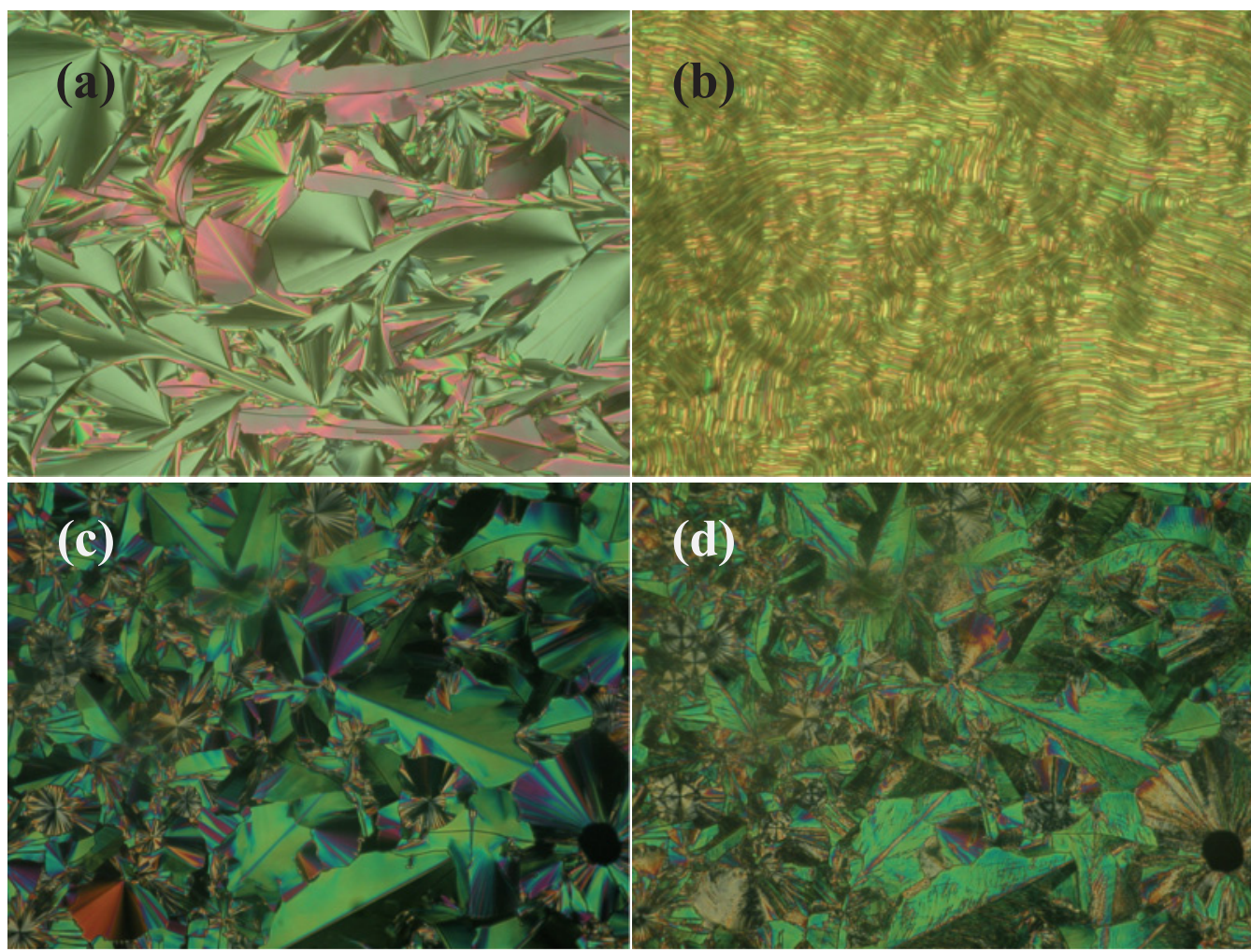

FIG. 3. (Color online) Texture of the $B_{1}$ phase of IP 31 in (a) a virgin sample and (b) under an electric field of $15 \mathrm{~V} / \mu \mathrm{m}$. The photomicrographs (c) and (d) correspond to NG126 under the same conditions as (a) and (b), respectively. (Width of the photomicrographs: $240 \mu \mathrm{m}$.)

mesophase under a low-frequency ac field [17]. However, when applying the electric field within the $\mathrm{B}_{1}$ phase, only an undefined grainy texture is obtained. The x-ray measurements clearly confirm the field-induced transition even well inside the $\mathrm{B}_{1}$ phase. In addition, the reversibility of the phase transition was also verified since after electric field removal the diffraction pattern corresponding to the columnar phase was restored in spite of the fact that no texture changes could be observed.
Figure 4(b) shows the x-ray pattern of NG126 at $133{ }^{\circ} \mathrm{C}$ without electric field (solid circles). Three peaks are clearly visible in the displayed angular range. Peak angles are compatible with the results presented in [22]. However, in that work the exposure times were longer and additional reflections were observed. The x-ray pattern was indexed on the basis of a centered oblique lattice. Following this indexation scheme our peaks correspond to (11), (02), and (22) reflections.
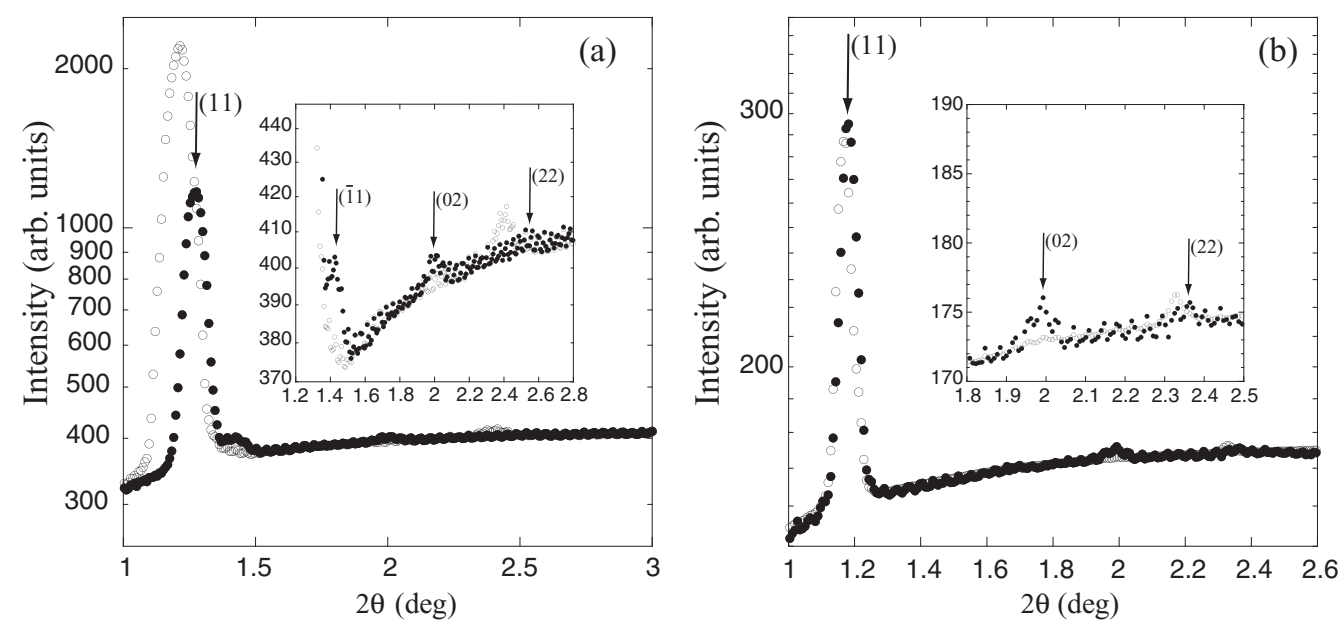

FIG. 4. X-ray scattering intensity versus $2 \theta$ of (a) IP31 at $145{ }^{\circ} \mathrm{C}$ and (b) $\mathrm{NG} 126$ at $133{ }^{\circ} \mathrm{C}$ obtained by azimuthal integration of the $2 \mathrm{D}$ $\mathrm{x}$-ray diffraction diagram. Solid circles were measured without electric field and open circles under 14 and $19 \mathrm{~V} / \mu \mathrm{m}$, respectively. In the insets small peaks are magnified. Only the peaks of the $\mathrm{B}_{1}$ phases are indexed. 

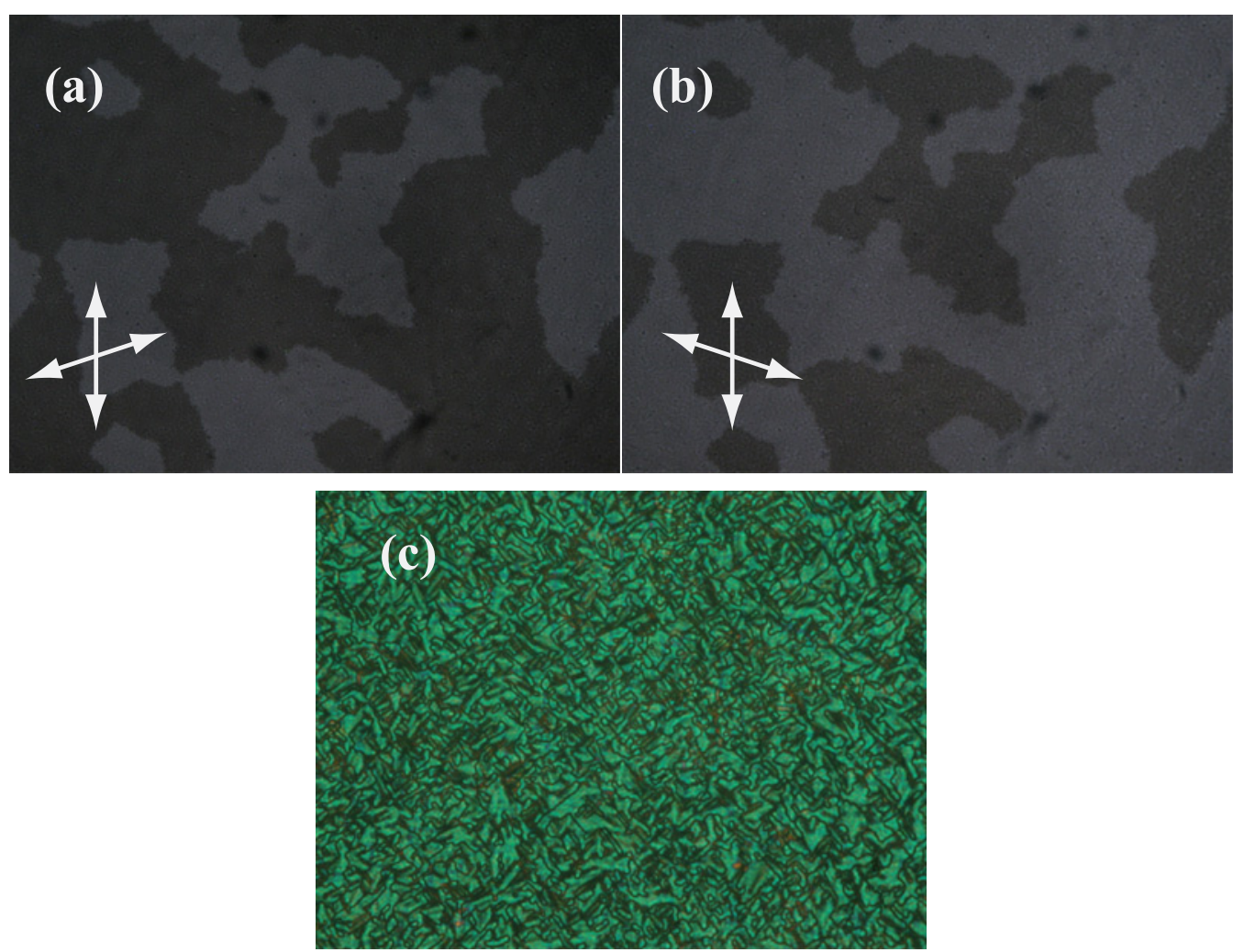

FIG. 5. (Color online) (a) and (b) DC texture of the NG75 after having applied an electric field of $10 \mathrm{~V} / \mu \mathrm{m}$ and subsequent removal. White arrows represent the polarizers slightly uncrossed to observe the chirality domains. (c) The birefringent texture of the same compound under $10 \mathrm{~V} / \mu \mathrm{m}$. (Width of the photomicrographs: $240 \mu \mathrm{m}$.)

Under an electric field of $19 \mathrm{~V} / \mu \mathrm{m}$ only two peaks are observed (open circles), which can be indexed in terms of a lamellar structure ( $\mathrm{SmCP})$ as $(01)$ and (02). The $\mathrm{x}$-ray diagram clearly confirms the $\mathrm{B}_{1}$-SmCP transition since the (02) peak in the $\mathrm{B}_{1}$ phase disappears under electric field. Similarly to the case of IP31 the field-induced phase transition is reversible once the field is removed. This reversibility is not apparent by texture observations [6].

\section{X-RAY STUDY OF THE DC PHASE}

As previously mentioned, the DC phase presents shortrange smectic order but the structure is optically isotropic. Although the constituent molecules are achiral, the macroscopic structure presents definite chirality. This fact can be easily checked since the optical activity is clearly observable due to the absence of birefringence [see an example in Figs. 5(a) and 5(b)]. The connection between the structure and optical properties has been studied in Refs. [12,23-26].

Under an electric field, the DC state undergoes a phase transition to a macroscopically anisotropic mesophase [see Fig. 5(c)]. The textures and electro-optic behavior suggest that the field-induced phases are different variants of the SmCP family [11-13]. However, this point it is not completely clear, since, again, textures often present domains that are too small for a reliable identification of the mesophase.

Figure 6 shows the chemical structure and phase sequence of the materials studied. The synthesis of NG75 together with a preliminary phase characterization has been published in
Ref. [27] (the material was named 3/14 in that study). Details of the synthesis of PAT11 and NG126 are reported in [19].

In the case of NG75, the material only presents the DC phase after applying an electric field and its subsequent removal. Otherwise the material presents an optically anisotropic phase identified as SmCP [11,13]. Figures 5(a) and 5(b) show the

\section{NG75}

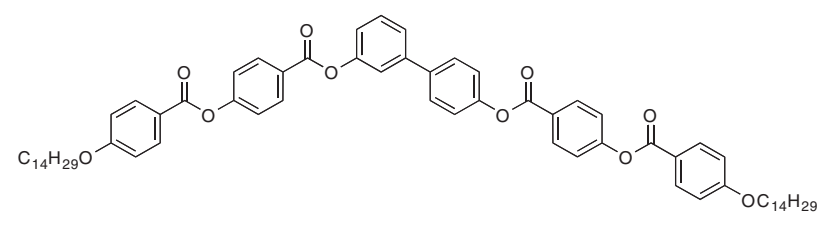

Iso $-157^{\circ} \mathrm{C}-\mathrm{DC}-68^{\circ} \mathrm{C}$ - Crystal

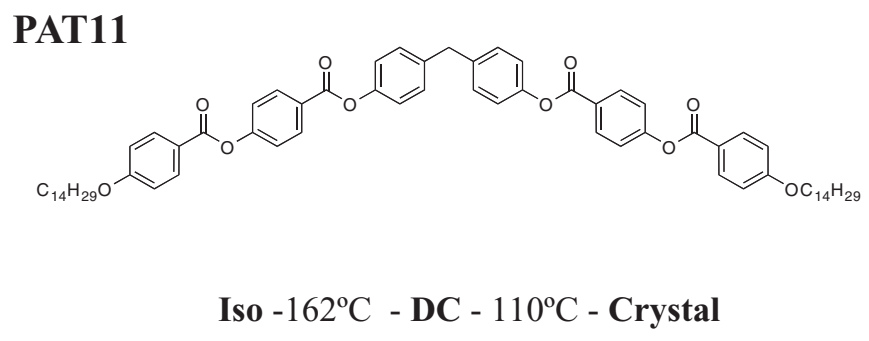

FIG. 6. Chemical structure and phase sequence of the studied compounds with DC phases. 


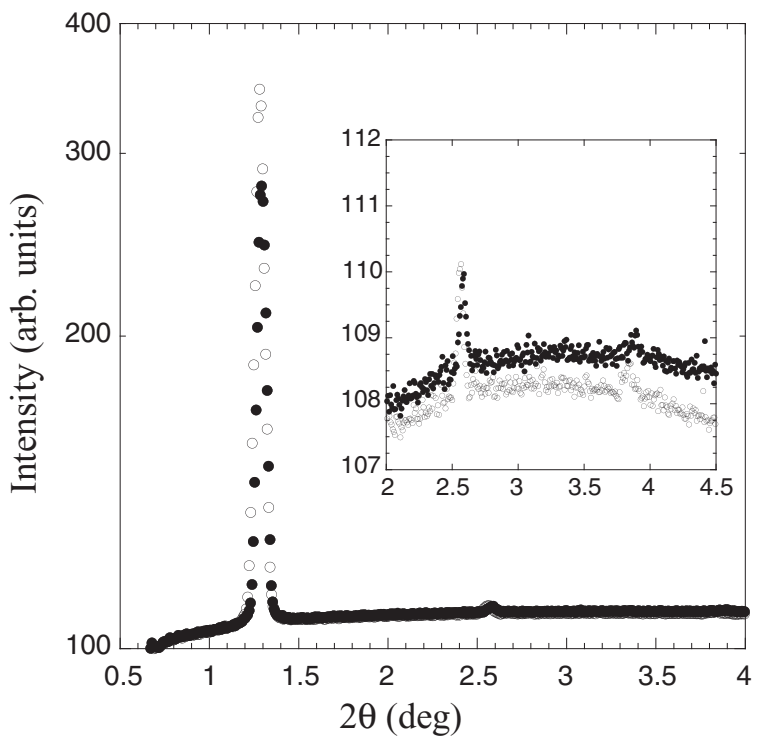

FIG. 7. X-ray patterns of NG75 at $130{ }^{\circ} \mathrm{C}$. Solid circles were measured without electric field and open circles under $14 \mathrm{~V} / \mu \mathrm{m}$. In the inset the higher-order harmonics are magnified.

isotropic texture of the DC phase and the chirality domains observed with slightly uncrossed polarizers. Under electric field a change is induced to a macroscopically anisotropic phase [Fig. 5(c)]. The texture presents small domains that seem to be characteristic of the SmCP phase. The switching mechanism between the DC to the anisotropic phase and in the opposite direction is abnormally fast in this compound $(<100 \mu \mathrm{s})$ [13].

Figure 7 presents the x-ray intensity versus $2 \theta$ of NG75 at a temperature of $130{ }^{\circ} \mathrm{C}$. Solid and open circles were measured under null and $14 \mathrm{~V} / \mu \mathrm{m}$ electric fields, respectively, and were obtained by azimuthal integration of the signal at the CCD camera. In both cases two harmonics of the main reflection (see inset) are clearly visible, which indicates that the structure is locally smectic. The diagrams are essentially the same, as it should be if the transition takes place between DC and SmCP. In order to find possible differences we examined the peak widths in both phases. Broader peaks are expected in the DC state according to the smaller correlation length. To check this point we carried out $2 \theta$ scans of the $(01)$ reflection in the same sample by using the point scintillation detector, with higher resolution than the CCD camera. The wave vector resolution in our experiment was $\delta q=1.6 \times 10^{-3} \AA^{-1}$ full width at half-maximum (FWHM). The resolution was checked with a silicon-powder capillary used as a standard. Figure 8(a) shows the (01) peak at $142{ }^{\circ} \mathrm{C}$. The squares were obtained after cooling down from the isotropic phase in the absence of field, whereas open circles are the results also at null field but after having applied a low-frequency $(1 \mathrm{~Hz})$ electric field of amplitude $6 \mathrm{~V} / \mu \mathrm{m}$ to provoke the appearance of the DC phase. Solid circles represent the intensity under an electric field of $6 \mathrm{~V} / \mu \mathrm{m}$. As can be seen, the peak intensity in this case is much higher due to a slight reorientation of the smectic layers, which have a tendency to form in a tighter angular distribution around the perpendicular to the substrate. Here, however, the important parameter is the FWHM. This was evaluated by fitting the peaks to a Lorentzian function after background subtraction. Solid lines in Fig. 8(a) represent the different fits. In all cases the width of the peaks is similar and it is resolution limited. The correlation length $\xi$ is related to the peak FWHM by $\xi=2 / \delta q$ [14]. Therefore a lower limit for $\xi$ is $\sim 125 \mathrm{~nm}$, i.e., about 30 layers in all cases. Although the result is compatible with the optical isotropy that the DC exhibits, the correlation length is long compared to other reported values $[14,28]$.

It is also interesting to point out that the angular position of the (01) peak decreases slightly under electric field. Although the effect is very small, it is clearly observable and reproducible, and the magnitude of the shift is proportional to the electric field. Figure 8(b) shows the layer spacing versus electric field. As can be seen, the dependence is linear but, contrary to what happens in a calamitic $\mathrm{SmC}^{*}$ phase, the
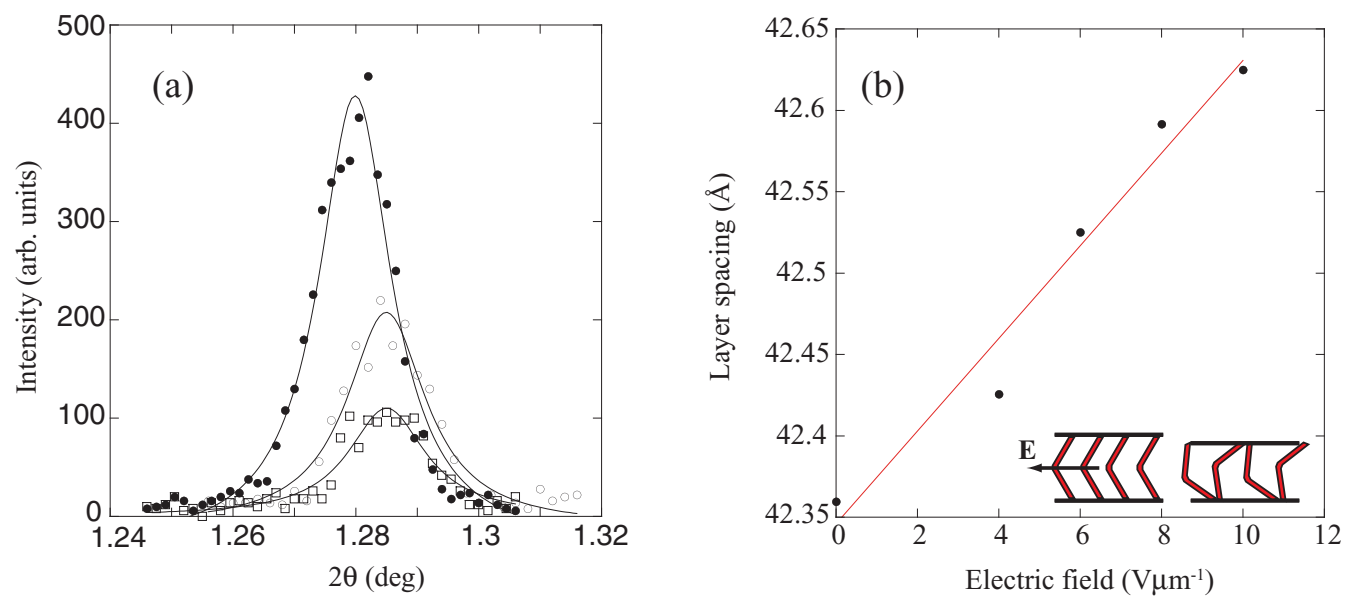

FIG. 8. (Color online) (a) (01) peak intensity versus $2 \theta$ of NG75 at $142{ }^{\circ} \mathrm{C}$. Squares were obtained in the virgin sample after cooling down from the isotropic phase $\left(\delta q=1.7 \times 10^{-3} \AA^{-1}\right)$, open circles correspond to null electric field but after having applied an electric field of $6 \mathrm{~V} / \mu \mathrm{m}$ to provoke the DC $\left(\delta q=1.8 \times 10^{-3} \AA^{-1}\right)$, and solid circles were obtained under $6 \mathrm{~V} / \mu \mathrm{m}\left(\delta q=1.6 \times 10^{-3} \AA^{-1}\right)$. The peaks are fitted to a Lorentzian function (continuous lines). (b) Layer spacing of the structure as a function of the applied electric field and sketch of a possible explanation of the layer expansion under field (for simplicity a null tilt is assumed). 

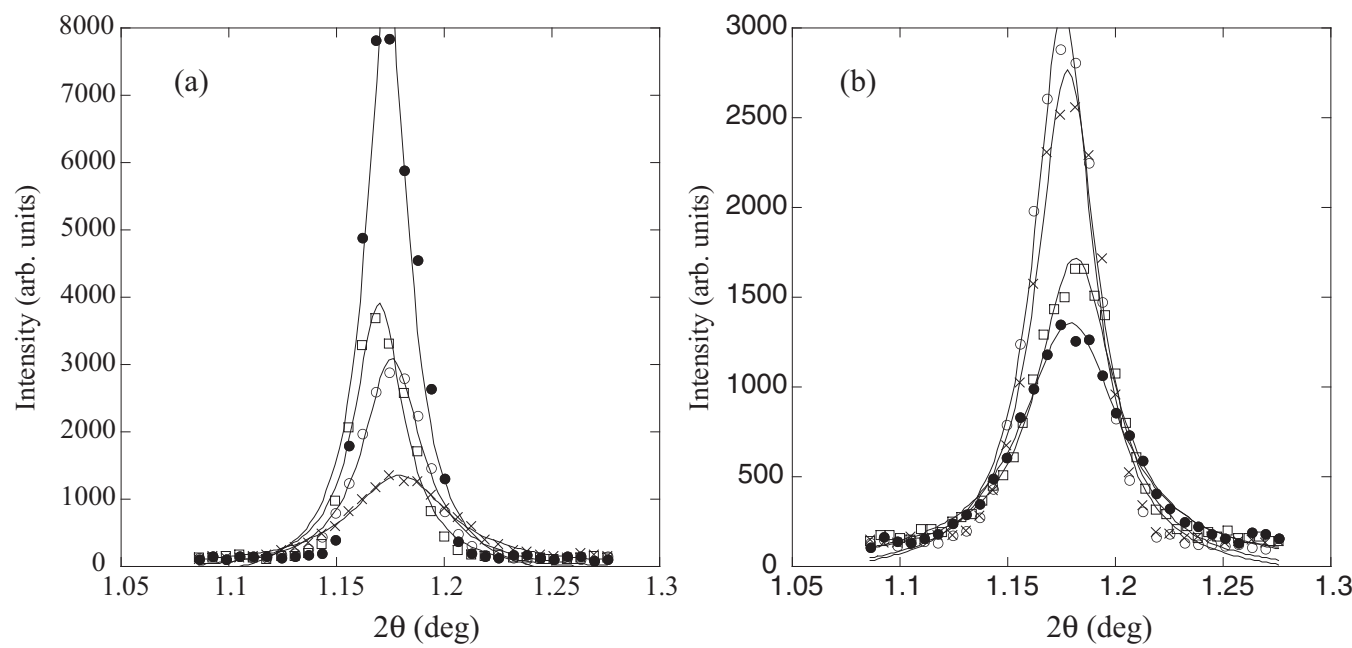

FIG. 9. (a) (01) peak intensity versus $2 \theta$ of PAT 11 at $150{ }^{\circ} \mathrm{C}$. Crosses were obtained in the virgin sample after cooling down from the isotropic phase $\left(\delta q=6.3 \times 10^{-3} \AA^{-1}\right)$, squares correspond to an electric field of $10 \mathrm{~V} / \mu \mathrm{m}\left(\delta q=3.1 \times 10^{-3} \AA^{-1}\right)$, solid circles to $12 \mathrm{~V} / \mu \mathrm{m}$ $\left(\delta q=2.9 \times 10^{-3} \AA^{-1}\right)$, and open circles just after field removal $\left(\delta q=3.8 \times 10^{-3} \AA^{-1}\right)$. (b) Relaxation process to the DC state after having applied an electric field of $10 \mathrm{~V} / \mu \mathrm{m}$. The $(01)$ peak is represented at different times: just after field removal (open circles, $\delta q=3.8 \times 10^{-3}$ $\AA^{-1}$ ), 2 min later (crosses, $\delta q=3.9 \times 10^{-3} \AA^{-1}$ ), $10 \mathrm{~h}$ afterwards (squares, $\delta q=5.1 \times 10^{-3} \AA^{-1}$ ). Solid circles correspond to the virgin sample in the DC phase $\left(\delta q=6.3 \times 10^{-3} \AA^{-1}\right)$. The peaks are fitted to a Lorentzian function in all cases (continuous lines).

slope is positive, i.e., the apparent molecular tilt decreases for increasing fields. The electroclinic effect in calamitic chiral smectic phases can be easily understood since the polarization and tilt are coupled order parameters and, thus, the induced polarization and tilt are proportional to each other. However, in the case of bent-core mesophases both quantities are not coupled and a true electroclinic effect should not be present. In any case, it must be pointed out that the observed effect is very small and is not necessarily linked to a tilt reduction. A plausible explanation could be that the leaning angular distribution of the molecules within the layers sharpens when the electric field is applied, in such a way that they become more tightly distributed along the null-leaning mean angular position [see the scheme in Fig. 8(b)]. Under this assumption the molecules would present the maximum length in the direction perpendicular to the smectic layers and therefore the layer spacing would increase.

The DC phase in the case of PAT11 presents a different behavior. On the one hand, the DC state appears spontaneously by cooling down the material through the clearing point. In addition, above a threshold electric field of $5 \mathrm{~V} / \mu \mathrm{m}$ a birefringent sandy texture is observed (see Fig. 3 of Ref. [12]). After field removal the DC state is restored after several hours. The small domain size prevents us from identifying the fieldinduced mesophase reliably. As in the previous compound, $\mathrm{X}$-ray results obtained by using the CCD detector confirmed the smectic nature of the phase both with and without electric field (the same pattern: a main reflection and two harmonics). Therefore the field-induced phase also belongs to some variant of the SmCP phase.

We studied the correlation length of the smectic ordering also by measuring the width of the diffraction peaks under different electric fields. In this material the weak signal required a slight opening of the diffractometer slits and the experimental resolution was $\delta q=3.0 \times 10^{-3} \AA^{-1}$. Figure 9(a) shows the (01) peak intensity versus $2 \theta$ at $150{ }^{\circ} \mathrm{C}$ in the virgin sample without field, under field, and just after field removal. The FWHM is $\delta q=6.3 \times 10^{-3} \AA^{-1}$ in the virgin sample and it is near resolution limited in the other cases. Deconvoluting the experimental resolution, a correlation length for the smectic order of $\sim 40 \mathrm{~nm}$ is obtained in the DC phase. This compound has been recently studied by Chen et al. using X-ray diffraction, freeze fracture transmission electron microscopy, and transmission light microscopy [28]. The correlation length found in that work agrees with our result. Just after field removal the layer-ordering correlation is similar to that of the phase under field. The result is in agreement with what is expected by texture observation, which reveals that the birefringent texture is maintained for a few hours once the field is removed. X-ray results of the relaxation process to the DC state are shown in Fig. 9(b). The FWHM of the peaks range from a minimum $\left(\delta q=3.8 \times 10^{-3} \AA^{-1}\right.$, near the experimental resolution) just after field removal to a maximum $\left(\delta q=5.1 \times 10^{-3} \AA^{-1}\right)$ after $10 \mathrm{~h}$. This indicates that $>10 \mathrm{~h}$ are required to restore the DC state completely.

Similarly to the previous case, angular peak positions shift to lower angles when increasing the electric field. In this case the layer spacing is also proportional to the electric field and has a similar magnitude to that found in NG75. This phenomenon could occur generally in bent-core smectic phases.

In summary, the main difference found between the two studied materials is the magnitude of the correlation length in the DC phases. In NG75 x rays do not reveal any difference between DC and SmCP phases. In contrast, peaks clearly sharpen under field in PAT11. Though we do not know why the $\xi$ values are so different, it is interesting to point at a possible relationship between this fact and the different ways of growing and switching dynamics of both DC phases. The investigation of this issue requires the analysis of more materials. 


\section{CONCLUSIONS}

Our x-ray measurements show that the structures induced by an electric field from the $\mathrm{B}_{1}$ and $\mathrm{DC}$ phases are truly SmCP. Since the field aligns the molecular dipoles, this means that the subsequent local packing of the bent-core molecules promotes the formation of flat smectic layers. The phase transitions are reversible in all cases, though the times to return to the ground state once the field is suppressed are highly variable, especially in the compounds with DC phases. In those materials an increase of the layer spacing as a function of the applied field has been noticed.

\section{ACKNOWLEDGMENTS}

This research was supported by MICINN-FEDER of SpainUE (Contracts No. MAT2008-06522-CO2, No. MAT200914636-CO3, and No. PI201060E013), the Aragón Government (E04), and the Basque Country Government (Project No. GIC10/45). The authors acknowledge the CSIC for financial support and for the provision of synchrotron radiation facilities. We would also like to thank the SpLine staff for their assistance in using beamline BM25B-SpLine for the X-ray diffraction experiments. One of us (N.G.) gratefully acknowledges the Juan de la Cierva and JAE-DOC contract programs.
[1] G. Pelzl, S. Diele, and W. Weissflog, Adv. Mater. 11, 707 (1999).

[2] R. Amaranatha Reddy and C. Tschierske, J. Mater. Chem. 16, 907 (2006).

[3] H. Takezoe and Y. Takanishi, Jpn. J. Appl. Phys. 45, 597 (2006).

[4] Y. Takanishi, T. Izumi, J. Watanabe, K. Ishikawa, H. Takezoe, and A. Iida, J. Mater. Chem. 9, 2771 (1999).

[5] J. Ortega, M. R. de la Fuente, J. Etxebarria, C. L. Folcia, S. Díez, J. A. Gallastegui, N. Gimeno, M. B. Ros, and M. A. Pérez-Jubindo, Phys. Rev. E 69, 011703 (2004).

[6] C. L. Folcia, J. Etxebarria, J. Ortega, and M. B. Ros, Phys. Rev. E 74, 031702 (2006).

[7] J. Kirchhoff and L. S. Hirst, Appl. Phys. Lett. 90, 161905 (2007).

[8] Y. Takanishi, M. Toshimitsu, M. Nakata, N. Takada, T. Izumi, K. Ishikawa, H. Takezoe, J. Watanabe, Y. Takahashi, and A. Iida, Phys. Rev. E 74, 051703 (2006).

[9] J. Kirchhoff and L. S. Hirst, Phys. Rev. E 82, 031701 (2010).

[10] J. P. Bedel, J. C. Rouillon, J. P. Marcerou, M. Laguerre, M. F. Achard, and H. T. Nguyen, Liq. Cryst. 27, 103 (2000).

[11] J. Etxebarria, C. L. Folcia, J. Ortega, and M. B. Ros, Phys. Rev. E 67, 042702 (2003).

[12] J. Ortega, C. L. Folcia, J. Etxebarria, N. Gimeno, and M. B. Ros, Phys. Rev. E 68, 011707 (2003).

[13] J. Martínez-Perdiguero, I. Alonso, C. L. Folcia, J. Etxebarria, and J. Ortega, Phys. Rev. E 74, 031701 (2006).

[14] L. E. Hough, M. Spannuth, M. Nakata, D. A. Coleman, C. D. Jones, G. Dantlgraber, C. Tschierske, J. Watanabe, E. Körblova, D. M. Walba, J. E. Maclennan, M. A. Glaser, and N. A. Clark, Science 325, 452 (2009).

[15] I. Alonso, J. Martínez-Perdiguero, J. Ortega, C. L. Folcia, and J. Etxebarria, Liq. Cryst. 34, 655 (2007).

[16] G. R. Castro, J. Synchrotron Radiat. 5, 657 (1998).
[17] C. L. Folcia, I. Alonso, J. Ortega, J. Etxebarria, I. Pintre, and M. B. Ros, Chem. Mater. 18, 4617 (2006).

[18] I. C. Pintre, N. Gimeno, J. L. Serrano, M. B. Ros, I. Alonso, C. L. Folcia, J. Ortega, and J. Etxebarria, J. Mater. Chem. 17, 2219 (2007)

[19] N. Gimeno, J. Barberá, J. L. Serrano, M. B. Ros, M. R. de la Fuente, I. Alonso, and C. L. Folcia, Chem. Mater. 21, 4620 (2009).

[20] N. Vaupotic, M. Copic, E. Gorecka, and D. Pociecha, Phys. Rev. Lett. 98, 247802 (2007).

[21] The cell parameters differ somewhat from those of Ref. [22]. This is not strange since it has been found that the confinement conditions of the sample (glass cells versus drops) can even give rise to a different phase sequence in this material.

[22] J. Martínez-Perdiguero, J. Etxebarria, C. L. Folcia, J. Ortega, N. Gimeno, and M. B. Ros, Phys. Rev. E 82, 041706 (2010).

[23] L. E. Hough and N. A. Clark, Phys. Rev. Lett. 95, 107802 (2005).

[24] L. E. Hough, C. Zhu, M. Nakata, N. Chattham, G. Dantlgraber, C. Tschierske, and N. A. Clark, Phys. Rev. Lett. 98, 037802 (2007).

[25] J. Etxebarria, C. L. Folcia, and J. Ortega, Phys. Rev. Lett. 101, 079801 (2008).

[26] L. E. Hough, C. Zhu, M. Nakata, N. Chattham, G. Dantlgraber, C. Tschierske, and N. A. Clark, Phys. Rev. Lett. 101, 079802 (2008).

[27] D. Shen, A. Pegenau, S. Diele, I. Wirth, and C. Tschierske, J. Am. Chem. Soc. 122, 1593 (2000).

[28] D. Chen, Y. Shen, C. Zhu, L. E. Hough, N. Gimeno, M. A. Glaser, J. E. Maclennan, M. B. Ros, and N. A. Clark, Soft Mater. 7, 1879 (2011). 